\title{
Health psychology in primary care: recent research and future directions
}

This article was published in the following Dove Press journal:

Psychology Research and Behavior Management

3I May 20I I

Number of times this article has been viewed

\author{
Stephen Thielke' \\ Alexander Thompson ${ }^{2}$ \\ Richard Stuart ${ }^{3}$ \\ 'Psychiatry and Behavioral Sciences, \\ University of Washington, Geriatric \\ Research, Education, and Clinical \\ Center, Puget Sound VA Medical \\ Center, Seattle, WA, USA; ${ }^{2}$ Group \\ Health Cooperative, Seattle, WA, USA; \\ ${ }^{3}$ Psychiatry and Behavioral Sciences, \\ University of Washington, Seattle, \\ WA, USA
}

\begin{abstract}
Over the last decade, research about health psychology in primary care has reiterated its contributions to mental and physical health promotion, and its role in addressing gaps in mental health service delivery. Recent meta-analyses have generated mixed results about the effectiveness and cost-effectiveness of health psychology interventions. There have been few studies of health psychology interventions in real-world treatment settings. Several key challenges exist: determining the degree of penetration of health psychology into primary care settings; clarifying the specific roles of health psychologists in integrated care; resolving reimbursement issues; and adapting to the increased prescription of psychotropic medications. Identifying and exploring these issues can help health psychologists and primary care providers to develop the most effective ways of applying psychological principles in primary care settings. In a changing health care landscape, health psychologists must continue to articulate the theories and techniques of health psychology and integrated care, to put their beliefs into practice, and to measure the outcomes of their work.
\end{abstract}

Keywords: health psychology, primary care, integrated care, collaborative care, referral, colocation

\section{Introduction}

In this article we appraise recent research findings related to health psychology and mental health in primary care. We hope that our synthesis of the research will be useful for psychologists working in and out of primary care, in addition to anyone engaged or interested in the provision of behavioral health care in primary medical settings. Some of our conclusions have implications for researchers and policy-makers.

We conducted a general review of health psychology by reading recent books and journals about this topic. This yielded numerous articles related to primary care. We then conducted MEDLINE and Google Scholar searches for combinations of the terms "health psychologist", "health psychology", "primary care", "general practice", "family practice", and "family medicine". After sorting and reading these, we delineated main themes about the practice of health psychology in primary care, and conducted additional searches in order to develop the context. We did not aim to present a comprehensive review of the field, but rather to identify and develop key issues and to make useful suggestions for future research.

\section{Terminology and context}

There is no official definition for what a health psychologist is or does. In 1982, Matarazzo defined health psychology as an aggregate field in psychology, involving 
educational, scientific, and professional contributions, and accomplishing a variety of ends: "the promotion and maintenance of health, the prevention and treatment of illness, the identification of etiologic and diagnostic correlates of health, illness, and related dysfunction and to the analysis and improvement of the health care system and health policy formation". ${ }^{1}$ A more recent analysis streamlines the definition as "an interdisciplinary field concerned with the application of psychological knowledge and techniques to health, illness and health care". ${ }^{2}$ This broad definition is mirrored in the description of the field from the American Psychological Association (APA): "Health psychologists participate in health care in a multitude of settings including primary care programs, inpatient medical units, and specialized health care programs such as pain management, rehabilitation, women's health, oncology, smoking cessation, headache management, and various other programs". ${ }^{3}$ While there are no clear boundaries around the activities of health psychologists in these different care settings, recent research about health psychology in primary care has focused largely on mental health rather than physical health promotion, with particular attention to depression.

The exact disciplinary boundaries around health psychology are somewhat indistinct. Health psychologists typically hold a doctoral degree in psychology ( $\mathrm{PhD}$ or PsyD) and complete general clinical training in the field. Advanced training programs in health psychology exist at the postdoctoral level, but these are not a prerequisite for clinical work in the field. Board certification in health psychology is available through the American Board of Professional Psychology, although no additional licensure is required beyond that needed for the independent practice of psychology. The national professional organizations in psychology have divisions related to health psychology (Division 38 in the APA and the Division of Health Psychology in the British Psychological Association), but these do not have a single disciplinary focus, and much of their work relates to general psychological practice. Health psychology has been divided into four main domains: clinical health psychology, public health psychology, community health psychology, and critical health psychology (the last of these directed at promoting social justice and addressing health inequalities). We will focus exclusively on clinical health psychology, since the other areas have little relevance to health care delivery in primary care.

We discuss several models in which health psychologists work in primary care settings. These mainly focus on the identification and treatment of mental health conditions, rather than the promotion of physical health. The terms to characterize these models are subject to different interpretations; see Miller et $\mathrm{al}^{4}$ for a more detailed description. "Colocation" describes having psychologists work in the same physical location as primary care providers. It typically uses cross-referrals and may or may not involve structured systems for communication and interaction between primary care providers and health psychologists. Mental health and primary care providers may work independently in a model termed "coordinated care". The term "enhanced referral" may be used when some parts of the referral process are managed in primary care, such as scheduling appointments for, transportation to, and payment for mental health services, with the aim of reducing barriers to receiving them. "Collaborative care" is a broad term for the structured relationships between primary care and mental health providers. Often another designated staff member, such as a mental health care manager, serves as the responsible agent both for following up with patients and for communicating between primary care and mental health providers. "Integrated care" is sometimes used synonymously with "collaborative care" to refer to a more structured program for interaction between primary care and mental health providers. Both collaborative and integrated care typically use formal data tracking systems, monitoring patient symptoms and treatments, care plans, and communications with patients and between providers. ${ }^{5}$

Most of the research we reviewed occurred in the United States, with the remainder from European countries, especially the United Kingdom. American health care is mainly owned by private for-profit and both private and public nonprofit health systems, which receive payments from insurance and patients. Several large federal health systems, especially the Veterans Health Administration (VA), administer medical facilities and provide services, but these are available only to select patient populations. About $60 \%$ of insured Americans receive health insurance through their employer; government programs such as Medicare (for older adults) and Medicaid (for categorically needy and disabled adults and children) directly cover about $25 \%$ of the population. About $15 \%$ of the population has traditionally been uninsured, and although recent legislation has offered a plan to insure all Americans; it is currently the subject of court challenges. Major changes in the current American health care landscape may or may not happen. ${ }^{6}$

Health psychologists in America thus work within public health care systems (such as the VA), within managed care systems (such as Kaiser Permanente), or in private settings. 
In the first of these, they usually receive a salary; in the latter two they may either be salaried or bill insurers or patients for services provided, or some combination thereof.

\section{Past research}

It is impossible to summarize all the recent work about health psychology in primary care. One quarterly journal, Journal of Clinical Psychology in Medical Settings, has devoted itself to this theme since 1994, and other journals in both primary care and psychology contain dozens of relevant articles each year, eg, Health Psychology, Journal of Health Psychology, British Journal of Health Psychology, and Applied Psychology: Health and Well-Being. At least seven books with comprehensive overviews have been published within the last decade..$^{2,7-12}$ They focus largely on practical issues related to establishing an effective practice, and to specific populations and health problems. Many include "how-to" checklists about establishing a health psychology practice in primary care, such as these key factors: 1) get your foot in the door; 2) be a team member (see all patients referred; view referring physicians as your primary customers; communicate well); 3) build key relationships (sit in with physician colleagues; act like a primary care provider; assist coworkers); 4) persist in marketing psychology services (designate a "problem of the week"; conduct daily check-ins); 5) be available; 6) learn the primary care culture (adopt the primary care pace; adopt a "population health" perspective; give prompt, succinct feedback); 7) attend to ethical issues; and 8) plan around financial issues. ${ }^{8}$ Instead of such tips for running a successful health psychology practice, we will focus on research around how health psychology functions in the current health care system, what evidence exists for its outcomes in primary care, what challenges exist, and how future research can advance the field.

\section{Unchanged factors in the health care landscape}

Despite numerous innovative interventions that seek to improve mental health care, the basic process and context of care have been mainly unchanged over the last several decades. Patients have the same psychological problems and needs; there is no evidence of significant changes in core psychopathology. Primary care remains the main location for mental health care in the US, and may even have expanded its scope as the use of psychotropic drugs has increased..$^{13}$ There has similarly been little change in the barriers limiting the receipt and provision of mental health care in primary or specialized settings. Many patients with serious mental illness do not receive any type of care; in 2004 it was estimated that only $37 \%$ of those with a mental illness received treatment for it. ${ }^{14}$ Primary care providers often fail to recognize mental health conditions; more than half of depressed patients in primary care were not diagnosed..$^{15}$ Of those referred for mental health treatment, about one-third failed to make their first appointment. ${ }^{16}$ Among the longstanding barriers to keeping appointments are the stigma of obtaining help, negative evaluations of therapy in general, and the time constraints, unavailability, and cost of interventions. ${ }^{17} \mathrm{Of}$ those who do start treatment, between half and two-thirds of patients terminate before they achieve even "minimally adequate treatment". ${ }^{18}$ The current mental health system thus fails at multiple levels: identifying patients with mental health problems, referring them for appropriate care, assuring follow-through, and achieving desired outcomes. ${ }^{19,20}$

\section{The effectiveness of health psychology interventions}

Health psychology, given its focus and setting, seems a natural solution to many of these persistent problems with mental health care delivery. We summarize some of the ways that health psychology has been shown to influence patient-level outcomes in primary care settings, and delineate how health psychology can impact physical as well as mental health. We then analyze evidence from recent studies about effectiveness and cost-effectiveness.

\section{Contributions of health psychology in primary care}

The benefits of having health psychologists in primary care settings have been formulated in a number of different ways, related either to the health psychologists' direct patient contact or their support of clinicians in identifying and managing mental health conditions. A recent exploration of integrated primary care identified nine such domains, some quite pragmatic and others general, including enhanced screening, unified treatment plans, phone follow-ups, immediate access, and common medical records. ${ }^{4}$ Although the specific effects of each of these factors have not been elucidated, earlier research has established the general efficacy of psychological interventions in enhancing primary care treatments of mental health. Most of the studies have used carefully organized interventions conducted by or in collaboration with academic centers. The positive outcomes in these are manifold: better patient retention, higher treatment adherence rates, improved outcomes, and cost savings. ${ }^{21-23}$ Because many of these studies happened about a decade ago and are summarized 
in most books and articles, we will not tabulate their results; Bluestein and Cubic's recent article ${ }^{24}$ gives a clear summary. A recent article suggests that the research around health psychology interventions has moved past the "discovery" and "refinement" stages, in which approaches were initiated, tested, and streamlined, to one of "dissemination", in which programs that are known to be effective can be more widely adopted by health care systems, and their utility demonstrated in real-world settings. ${ }^{25}$

\section{Physical health promotion}

An important theme from earlier work about health psychology in primary care is that psychological interventions have benefits not only for treating mental health conditions, but also for improving health. Addressing both physical and mental health problems in primary care allows sharing of diagnostic information, determining treatment plans, and monitoring the patient's overall health status rather than specific symptoms. ${ }^{26}$ Patients suffering from mental health conditions often present with physical symptoms and use more health care services, and improving mental health services can ensure that they receive the most accurate diagnoses and effective treatments for both. ${ }^{27,28}$ Research showing benefits of integrated care interventions on physical symptoms such as pain ${ }^{29}$ and chronic diseases such as diabetes ${ }^{30}$ highlight the importance of delivering psychological interventions in primary care settings.

Despite the important bidirectional association between physical and mental health, most of the recent research about health psychology interventions in primary care has focused on the identification and management of mental health conditions. The discipline of health psychology often plays a key role in the management of physical health problems, but usually in specialized clinical settings (as described above around the domains in which health psychologists work, such as inpatient medical wards or oncology clinics). Almost all of the recent effectiveness research we analyzed derives from integrated or collaborative care programs, which are geared at mental health conditions.

\section{Recent meta-analyses of outcomes}

The aforementioned evidence for the benefits derived from psychological interventions in primary care seems overwhelmingly positive, yet recent research has generated mixed results around effectiveness and cost-effectiveness. A recent Cochrane review of counseling in primary care found that despite higher patient preference and satisfaction, "in the long term, counseling is not any better than
GP care", and that "counseling does not seem to reduce overall health care costs". ${ }^{31}$ Another meta-analysis about the cost-effectiveness of psychological treatments for depression in primary care found that "the cost-effectiveness of counseling in comparison with usual care and antidepressant therapy is yet to be established". ${ }^{32}$ These findings must be qualified by the fact that counselors in this review were not necessarily health psychologists, although they applied the principles of health psychology.

A meta-analysis of ten randomized controlled trials for psychological interventions for depression in primary care found advantages over usual care, but no differences between psychological interventions and antidepressant treatment. ${ }^{33}$ Psychological interventions were defined by the use of an explicit psychological orientation (such as cognitivebehavioral or psychodynamic frameworks), scheduling a standardized number of sessions, and administration by trained practitioners, including clinical psychologists, general practitioners, psychiatrists, counselors, social workers, nurses, or volunteers. The combining of all types of practitioners into one group limits how much the results can be generalized to health psychology (about half of the trials used psychologists to administer the psychological intervention). Another recent meta-analysis of consultation-liaison mental health care in the management of depression in primary care suggested that it is no more effective than usual care, although this consult-liaison model is somewhat different than that applied by most health psychologists. ${ }^{34} \mathrm{~A}$ meta-analysis of collaborative care interventions found that collaborative care was more effective than standard care, but that the outcomes were driven largely by medication adherence and care manager variables, and that the addition of brief psychotherapy did not improve outcomes. ${ }^{35}$ These results should not be interpreted to suggest that psychological interventions in primary care settings are not effective or cost-effective; rather they show the absence of high-quality evidence from controlled trials of specific health psychology interventions.

Another area in which quality evidence is lacking is in real-world effectiveness. Although the main results around outcomes have come from small and carefully conducted studies, usually completed at or managed by academic medical centers, the real test of real-world effectiveness would come from measured benefits in large health care systems. Yet there are no carefully-conducted evaluated studies of pragmatic trials conducted in usual practice conditions, sometimes called "natural interventions". ${ }^{36}$ The VA's initiative to increase the use of health psychology in primary care has been characterized, with plans to study it, ${ }^{37}$ 
but without demonstrated patient-level benefits compared to other approaches. Robinson and Strosahl described their successes and challenges in introducing integrated behavioral health programs in several large health care systems, including Kaiser Permanente and the Air Force and Navy; ${ }^{25}$ but to our knowledge they have not published results about effectiveness or cost-effectiveness of these initiatives. Anecdotal reports of the Air Force project have been published, without outcome data. ${ }^{38}$ This lack of empirical evidence does not suggest that these programs do not yield benefits, but rather emphasizes the need for additional pragmatic research.

\section{Current and future challenges}

Beyond the absence of evidence-based outcomes in controlled research settings or real-world treatment settings, several additional key challenges remain to the practice of health psychology in primary care. We will discuss four that have the most immediate relevance. First, how successfully has health psychology penetrated primary care treatment settings? Second, how do integrated care models use the services of health psychologists? Third, how can health psychologists adapt to pressures around financial accountability in clinical care? Fourth, how does the rapid expansion of psychotropic medication prescribing influence the provision of psychological interventions?

\section{What is the penetration of health psychology in primary care?}

Patients prefer to receive mental health treatment in primary care settings, ${ }^{39}$ and are more likely to attend follow-up there than in specialty mental health settings. ${ }^{40}$ With the goal of maximizing the number of patients who can be treated in primary care settings, it seems important to know how many primary care practices now involve health psychologists, yet it has been very difficult to estimate this figure. It has been suggested by Gatchel and Oordt (2006) that health psychologists are a common part of primary care clinics: "Primary care has been a professional home for many psychologists over the past 30 years".${ }^{8}$ Conversely, when writing about integrating psychology within primary care James (2006) notes, "The primary care setting offers a mostly new and exciting opportunity for clinical psychology". ${ }^{41}$ There is no quantitative information to support these discussions, and to our knowledge, research has not clarified how many primary care clinics use health psychology interventions or integrated care models. A 2010 employment survey from the APA (obtained through personal communication) found that about $30 \%$ of psychologists report involvement in primary or integrated health care, but this does not quantify the overall prevalence of health psychologists working in these settings.

Data exist for one large health care system, the VA. Since 2004, the VA has worked to increase psychological services in primary care. By 2008 it had plans to fund 509 primary care programs and 310 psychology staff positions; around 100 had been hired. ${ }^{37}$ The denominator for these settings is difficult to calculate, but is roughly 1000 primary care clinics. This suggests that about one-third to one-half of the VA's primary care clinics have incorporated psychological services. An analysis of the VA's program in 2010 did not provide a more detailed estimate of the overall penetrance, and focused instead on integration projects. ${ }^{42}$ The VA has been a case of extremely wide adoption, able to apply large resources within an organized and delimited system, and we speculate that a smaller fraction of primary care practices across the US, especially private practices, routinely involve health psychologists. With the existing data it seems impossible to estimate the prevalence of health psychologists or psychological interventions in primary care clinics in the US or other countries. This information would be important for estimating overall impact and planning future policies.

\section{The role of the health psychologist in integrated care}

As Patricia Robinson aptly notes, "Next to the word 'love', the word 'integration' is among the most frequently used and abused words in the English language. Almost any activity involving two or more people is now labeled integrated". ${ }^{25}$ The vast majority of the novel mental health initiatives in primary care over the last decade have involved some form of integrated or collaborative care, in which primary care and mental health practitioners work together in a structured program to treat common mental health conditions. Numerous studies have shown this form of treatment to be significantly more effective and cost-effective than usual care (see Bluestein and $\mathrm{Cubic}^{24}$ and Miller et $\mathrm{al}^{4}$ for overviews). Integrated care has been described as "an essential part of the solution for our struggling American health care system". ${ }^{4}$

Yet a closer examination of integrated care models exposes a key challenge about the role of the health psychologist. While these programs integrate psychological interventions with primary care, many of the programs do not directly employ the services of health psychologists, especially in traditional roles such as receiving referrals and providing unstructured psychotherapy. Recent collaborative 
care models involve a structured set of relationships between the patient, primary care provider, consulting psychiatrist, and care manager (or “depression care coordinator") ${ }^{23}$ Care managers come from a variety of backgrounds, including nursing, social work, and psychology, and receive training about psychological principles, but often do not have advanced degrees in mental health fields. The health psychologists who do work as care managers spend a considerable part of their time not directly providing clinical care, performing actions such as checking in with patients by phone, documenting symptoms using tracking systems, discussing cases with the supervising psychiatrist, and facilitating medication changes. Other recent integrated care interventions that apply telephone-based screening, outreach, and care for depression seem to be effective in primary care, but they combine psychological treatments with medications and enhanced screening. ${ }^{43}$ Health psychology principles support the interventions, but sometimes without psychologists being materially involved in their traditional roles, and most of the interventions use pharmaceutical treatments as a key treatment option.

One might argue, in fact, that some integrated care models limit the involvement of health psychologists by having care managers carry out the evaluation and psychological treatments. For example, care managers in integrated care programs receive training in and deliver brief forms of psychotherapy such as problem-solving treatment, even though most of them are from disciplines other than psychology, especially nursing and social work. ${ }^{44}$ Many patients in these programs never come in contact with a health psychologist. The emphasis on psychotropic medications may also be seen as a means of minimizing the use of the health psychologist in providing therapy. It is thus difficult to disentangle the benefits of the psychological component from the larger intervention or the prescription of medications, or to compare its effectiveness with more traditional health psychology roles, such as enhanced referral or consultation-liaison.

Along the same lines, recent overviews argue that health psychologists have not adapted well to novel integrated care models. One finds, "Despite the negative impact of failing to make a transformation, most psychologists have not modified their practice and most training programs do not prepare psychologists to provide integrated care". ${ }^{24}$ Even though health psychologists and primary care providers generally share goals and wish to collaborate, they are held back from greater collaboration because of differences in education and training, clinical styles, reimbursement, and patient preferences. ${ }^{45}$ Another recent article speculates that health psychologists' failure to accommodate the financial realities of health care have stopped them from capitalizing on integrated care models: "America pays for health care, not psychosocial care, and all other professions rendering treatment... have taken advantage of the nation's evolution from a medical system to a health care system. As part of this health care system they are prospering, while psychotherapy is languishing". ${ }^{46}$ It posits that the barriers to better integration entail various factors such as "reluctance of mental health practitioners to give up solo practice, the 50-minute hour, and their traditional mode of practice", as well as "archaic training models that don't prepare psychologists to provide integrated care", and "the fact that our current third-party payer system is not constructed to meet the funding of this evolving system". Although little empirical data exist about how health psychologists have or have not adapted to integrated care, these observations suggest that some traditional psychology practices such as one-on-one 50-minute sessions may be discordant with recent integrated care models. As such, it appears that research around health psychology has in some cases appropriated positive outcomes from integrated care models, but these are not necessarily technically health psychology interventions, and may have limited roles for health psychologists.

\section{Increased financial accountability}

Almost imperceptibly, medicine in the United States has changed to accommodate financial goals. Providers must document what services they provided for specific diagnoses, all in standardized frameworks. The use of diagnostic codes (such as ICD-9) was driven not by clinical ends but by demands from payers, especially insurers, who have refused to pay for services unless the providers used codes and followed rules. Psychotherapy, which in the past may have enjoyed public recognition as an effective or even miraculous method of treatment, and which operated by its own rules, has increasingly become shackled by requirements for reimbursement. Mental health providers are being called upon to provide evaluative data showing cost-benefit and cost-effectiveness value. Third-party payers such as insurers, Medicaid, and Medicare use these as the standards for determining the utility and thus the value of reimbursing different types of treatment. An even more direct linkage between these principles and payments occurs in "pay for performance" models. ${ }^{47}$ Rather than paying providers for the amount of time they spend doing what they do, advocates of pay for performance want to base reimbursement on how much they accomplish. ${ }^{48}$ Performance-based reimbursement 
is growing rapidly and is now used by more than half of the commercial health maintenance organizations. ${ }^{49}$

The need to justify treatments and to document outcomes using reimbursement frames may seem consonant with the outcome-based goals of health psychology in primary care, but it can also complicate efforts to work collaboratively in shared settings, or to provide treatments which third-party payers are reluctant to fund. For instance, patients seen by health psychologists in primary care are almost by necessity "double-treated": they see both their main primary care provider, as well as a health psychologist, and often for the same underlying condition. This is especially true if the primary care provider prescribes a psychotropic medication and the health psychologist offers therapy. From the providers' perspectives, there may be manifest benefits in such a system (as documented in the recent books on the subject, cited above), but the payer may wonder why they are paying two people to treat one condition.

Health psychologists in primary care thus seem obligated to provide assurances to payers that the time invested in therapy is well spent, and must use standardized codes to do so. Efforts to justify the provision of psychotherapy in primary care require not only evidence that it is a scientifically-based, outcomes-driven practice, but also that it is a valuable supplemental service to primary care treatments (and vice versa). Yet there are several challenges around the documentation of outcomes from the clinical activities of health psychologists. First, the relationships between providers can be complex and overlapping, especially in integrated care models. Various providers may have direct and indirect roles in patient treatment decisions and direct patient contact. The costs and savings related to specific providers in such systems can be hard to quantify. Second, even in more traditional consultation models, the patient typically continues to see the primary care provider, who may prescribe a treatment such as psychotropic medications. As a result the specific factors behind patient outcomes can be hard to untangle. If the patient improves, is it a result of the antidepressant or the psychological intervention? Third, part of the anticipated benefit of involving health psychologists is the reduction in unnecessary medical evaluation or treatment, which can be very hard to measure objectively because it requires estimating a cost for each patient had they not received the psychological intervention. Addressing these challenges, and generating meaningful data around economic benefits, will likely require carefully conducted research involving treatment and comparison groups, perhaps with a factorial design (such as with or without a health psychologist's involvement).

\section{Increased use of psychotropic drugs}

Forty years ago it looked as though the survival of psychotherapy depended upon how well it could incorporate the rapidly developing advances in neuroscience. ${ }^{50}$ There have indeed been remarkable strides in neuroscience, but, coupled with advances in industrial neurochemistry and nearly unrestrained marketing of medications, they have led to a massive explosion of psychotropic drugs rather than a fortification of psychotherapy. In 1998, 40\% of patients with a mental health condition received a psychotropic medication; by 2007 it was $75 \%$, a total of about 23 million Americans. ${ }^{51}$ Use of psychotropic drugs has been steadily rising among all groups, ${ }^{52}$ including the elderly, ${ }^{53}$ adolescents, ${ }^{54}$ and children. ${ }^{55}$ Primary care has been the main growth area for these medications, and $70 \%$ of all psychotropics are now prescribed by primary care providers. ${ }^{41}$

As drug therapy has expanded, the use of psychological treatments has contracted. Over the last 10 years, referrals for psychotherapy have fallen by almost 50\%. ${ }^{56}$ During the same period, the percentage of patients for whom antidepressant medication was prescribed rose from $73 \%$ to $86 \%{ }^{19}$ A number of factors relevant to primary care contributed to this transition. Because of capitation contracts, managed care organizations had to pay for all of the psychotherapy their patients received but not all of the medications. Primary care physicians who delivered an increasing percentage of mental health services were better trained in medication management than psychotherapy. Even if they feel competent in delivering talk therapy, the time constraints of their busy practices encourage prescribing psychotropic medications instead. Marketing to prescribing providers grew at an exponential rate, proportionate to their profits from the sale of psychotropic drugs. ${ }^{13}$

Direct-to-consumer advertising also exploded during this time, and patients now often present to their physician asking to be prescribed a specific medication. ${ }^{57}$ These requests encourage providers to write a prescription when they might have thought that another form of therapy or drug would have been preferable. It takes far less time to comply with medication requests than to convince patients to take a different course. In addition, providers often feel better when they can "do something" by allowing patients to leave the session with a prescription.

Although we do not have room here to discuss all the complex issues around psychotropic drugs in primary 
care, and their effects on health psychologists, they clearly seem to have threatened provision of nonpharmacological treatments. Recently the mainstream medical community has begun to recognize this problem. A recent commentary in the Journal of the American Medical Association expressed considerable skepticism about the use of psychotropic drugs. They offered 25 principles, the first two of which are: "seek nondrug alternatives as first rather than last resort" and "treat underlying causes rather than solely treating symptoms". ${ }^{58}$ The importance of nonpharmacological interventions also becomes clear in recent evidence that patients prefer psychotherapy over medications. ${ }^{59,60}$ Added complexity comes from studies comparing psychotropic medications to psychotherapy, which have generally produced mixed results. ${ }^{61}$ It seems unlikely that any quick or major changes will happen in this domain in the near future, and psychotropic medications will likely continue to play a major role in primary care mental health treatment. Issues around psychotropic medications thus demand ongoing attention from health psychologists.

\section{Discussion}

We have attempted to provide a balanced assessment of recent developments in and challenges around health psychology in primary care. Our approach may seem somewhat pessimistic because it focused on challenges and absence of evidence rather than accomplishments. Yet we believe, based on our analysis of the literature, that both the perennial and novel challenges can be addressed, and that identifying them clearly is the first step. In the process of attending to system-level challenges and empirical data, we may have ignored the work of the many health psychologists who enact effective patient-centered care with every patient they see. Because of bias towards publishing data-driven studies, their work and positive outcomes may seem invisible. One should not interpret our findings, especially around lack of evidence of measurable benefit in some domains or in meta-analyses, as an attempt to detract from what health psychologists do in primary care. Rather they point out areas for further research and development, especially in demonstrating the effectiveness of health psychology within real-world clinic systems.

In focusing on the role of health psychologists in clinical work, we may also have understated some of the broader and less tangible benefits that they bring to primary care. Health psychologists have, through their ongoing activities, likely increased the awareness of mental health conditions among patients and providers, reduced the stigma of mental illness, challenged common assumptions about patients with mental health symptoms, and educated providers in various other disciplines. As described above, they have also worked to identify, understand, and treat the psychosocial factors involved in medical illness, and developed programs to encourage health-promoting lifestyle changes. Their work has also highlighted ethnic and psychological perspectives on illness, treatment methods, and the nature of outcomes, which can help providers understand the meanings patients ascribe to illness and health care. These sorts of contributions have not received much research attention recently, perhaps having been overshadowed by integrated care. For instance, a recent overview of training psychologists to work in primary care settings discusses only their clinical functions. ${ }^{62}$

Our synthesis of recent literature highlights some major gaps in knowledge, which can be addressed by future research. We suggest that researchers and policy makers focus on these questions, and that empirical evidence around them can help to improve the provision of mental health treatment in primary care.

1. Descriptive data on the prevalence of health psychology practices in primary care would greatly enhance the discussion. How many primary care clinics employ a health psychologist as part of their staff? How many have colocated health psychologists? How many use integrated care models? Once these data are collated, researchers can explore factors associated with uptake of various models of care, can evaluate outcomes, and can define regional variations or socioeconomic disparities. Such information would help organize future service delivery and policy.

2. As mentioned above, there are few carefully conducted studies examining how health psychologists have influenced primary care practice in large health care systems, as part of "natural interventions". The VA's primary care mental health initiative is being studied, and some research has tracked the effect of the VA's integration programs on diagnosis and utilization. ${ }^{42}$ Other systems that have used health psychologists in various roles can also provide relevant data. These evaluations should apply current methodologically sound principles in trial design. ${ }^{63}$

3. The exact role of the health psychologist in integrated (or collaborative) care is at times nebulous, with some interventions using them in nontraditional roles, and others using agents besides psychologists to deliver therapy. Evaluations showing the effectiveness of integrated care programs should account for the material contributions of health psychologists in the interventions. 
4. The two main challenges described above (greater financial accountability and increased prescription and marketing of psychotropic drugs) have and will continue to influence the practice of health psychology in primary care, and some research has indicated that health psychologists are not well prepared to adapt to them. Further exploration of approaches for adapting to these challenges is clearly warranted. For instance, reimbursement for the management of mental health problems by multiple providers (eg, the primary care provider prescribing medications, the health psychologist providing psychotherapy, and possibly a care manager for integrating care) may demand novel billing mechanisms. Ongoing advocacy efforts may be needed to promote psychotherapy as a viable and effective adjunct or alternative to psychotropic medications.

5. More research about the contributions of health psychologists to the practice improvement of other providers would help to characterize some of the apparently intangible benefits of this work, especially in the application of psychology research to common primary care conditions.

6. It seems certain that health psychology, primary care, and the overall health care landscape will continue to change in the near future, with new demands, pressures, and opportunities. To understand, adapt to, and respond to these changes, health psychology professionals will need to continue to articulate the theories and techniques of health psychology and integrated care, to put their beliefs into practice, and measure the results of their efforts.

In summary, recent research has shown how health psychology contributes to health promotion in primary care settings, but work remains around demonstrating its effectiveness and cost-effectiveness across different systems of care, and adapting it to longstanding and novel challenges.

\section{Disclosure}

No conflicts of interest were declared in relation to this paper.

\section{References}

1. Matarazzo JD. Behavioural health's challenge to academic, scientific and professional psychology. Am Psychol. 1982;37:1-14.

2. Marks DF, Murray M, Evans B, Willig C, Woodall C, Sykes CM. Health psychology: theory, research, and practice. 2nd ed. London, UK: Sage Publications; 2005.

3. Association AP. Becoming a health psychologist. Available at: http:// www.health-psych.org/AboutHowtoBecome.cfm. Accessed April 7, 2011.

4. Miller BF, Mendenhall TJ, Malik AD. Integrated primary care: an inclusive three-world view through process metrics and empirical discrimination. J Clin Psychol Med Settings. 2009;16:21-30.
5. Unutzer J, Choi Y, Cook IA, Oishi S. A web-based data management system to improve care for depression in a multicenter clinical trial. Psychiatr Serv. 2002;53:671-673, 678.

6. Glassock RJ. Health care reforms in America: perspectives, comparisons and realities. QJM. 2010;103:709-714.

7. Frank RG, McDaniel SH, Bray JH, Heldring M. Primary care psychology. Washington, DC: American Psychological Association; 2003.

8. Gatchel RJ, Oordt MS. Clinical health psychology and primary care: practical advice and clinical guidance for successful collaboration. Washington, DC: American Psychological Associaton; 2003.

9. James LC, O'Donohue WT. The primary care toolkit: practical resources for the integrated behavioral care provider. New York: Springer; 2008.

10. Hunter CL, Goodie JL, Oordt MS, Dobmeyer AC. Integrated behavioral health in primary care: step-by-step guidance for assessment and intervention. Washington, DC: American Psychological Association; 2009.

11. Haas LJ. Handbook of primary care psychology. Oxford: Oxford University Press; 2004.

12. Robinson PJ, Reiter JT. Behavioral consultation and primary care: a guide to integrating services. New York: Springer-Verlag; 2006.

13. Druss BG. The changing face of US mental health care. Am JPsychiatry. 2010;167:1419-1421.

14. Barry CL. Trends in mental health care. Issue brief (Commonw Fund). 2004:1-8.

15. Fernandez A, Saameno JA, Pinto-Meza A, et al. Burden of chronic physical conditions and mental disorders in primary care. $\mathrm{Br} J$ Psychiatry. 2010;196:302-309.

16. Zivin K, Pfeiffer PN, McCammon RJ, et al. "No-shows": who fails to follow up with initial behavioral health treatment? Am J Manag Care. 2009;15:105-112.

17. Mohr DC, Ho J, Duffecy J, et al. Perceived barriers to psychological treatments and their relationship to depression. J Clin Psychol. 2010;66:394-409.

18. Hansen NB, Lambert MJ. An evaluation of the dose-response relationship in naturalistic treatment settings using survival analysis. Ment Health Serv Res. 2003;5:1-12.

19. Mojtabai R, Olfson M, Sampson NA, et al. Barriers to mental health treatment: results from the National Comorbidity Survey Replication. Psychol Med. 2010:1-11.

20. Thielke S, Vannoy S, Unutzer J. Integrating mental health and primary care. Prim Care. 2007;34:571-592, vii.

21. Arean PA, Alvidrez J, Barrera A, Robinson GS, Hicks S. Would older medical patients use psychological services? Gerontologist. 2002;42: 392-398.

22. Katon WJ, Roy-Byrne P, Russo J, Cowley D. Cost-effectiveness and cost offset of a collaborative care intervention for primary care patients with panic disorder. Arch Gen Psychiatry. 2002;59:1098-1104.

23. Unutzer J, Katon W, Callahan CM, et al. Collaborative care management of late-life depression in the primary care setting: a randomized controlled trial. JAMA. 2002;288:2836-2845.

24. Bluestein D, Cubic BA. Psychologists and primary care physicians: a training model for creating collaborative relationships. J Clin Psychol Med Settings. 2009;16:101-112.

25. Robinson PJ, Strosahl KD. Behavioral health consultation and primary care: lessons learned. J Clin Psychol Med Settings. 2009;16:58-71.

26. Skultety KM, Zeiss A. The treatment of depression in older adults in the primary care setting: an evidence-based review. Health Psychol. 2006;25:665-674.

27. Karlin BE, Fuller JD. Meeting the mental health needs of older adults. Geriatrics. 2007;62:26-35.

28. Tai-Seale M, Bramson R, Drukker D, et al. Understanding primary care physicians' propensity to assess elderly patients for depression using interaction and survey data. Med Care. 2005;43:1217-1224.

29. Dobscha SK, Corson K, Perrin NA, et al. Collaborative care for chronic pain in primary care: a cluster randomized trial. JAMA. 2009;301: $1242-1252$. 
30. Katon WJ, Von Korff M, Lin EH, et al. The Pathways Study: a randomized trial of collaborative care in patients with diabetes and depression. Arch Gen Psychiatry. 2004;61:1042-1049.

31. Bower P, Rowland N, Mellor C, Heywood P, Godfrey C, Hardy R. Effectiveness and cost effectiveness of counselling in primary care. Cochrane Database Syst Rev. 2002;(1):CD001025.

32. Bosmans JE, Hermens ML, de Bruijne MC, et al. Cost-effectiveness of usual general practitioner care with or without antidepressant medication for patients with minor or mild-major depression. J Affect Disord. 2008;111:106-112.

33. Bortolotti B, Menchetti M, Bellini F, Montaguti MB, Berardi D. Psychological interventions for major depression in primary care: a meta-analytic review of randomized controlled trials. Gen Hosp Psychiatry. 2008;30:293-302.

34. Cape J, Whittington C, Bower P. What is the role of consultationliaison psychiatry in the management of depression in primary care? A systematic review and meta-analysis. Gen Hosp Psychiatry. 2010;32:246-254

35. Gilbody S, Bower P, Fletcher J, Richards D, Sutton AJ. Collaborative care for depression: a cumulative meta-analysis and review of longer-term outcomes. Arch Intern Med. 2006;166:2314-2321.

36. Research UNIfH. NIHR Health Technology Assessment Program. 2011; Available at: http://www.hta.ac.uk/about/index.shtml. Accessed April 7, 2011.

37. Zeiss AM, Karlin BE. Integrating mental health and primary care services in the Department of Veterans Affairs health care system. J Clin Psychol Med Settings. 2008;15:73-78.

38. Wilson PG. The Air Force experience: integrating behavioral health providers into primary care. In: Frank RG, McDaniel SH, Bray JH, Heldring M, editors. Primary care psychology. Washington, DC: American Psychological Association; 2004.

39. Chen H, Coakley EH, Cheal K, et al. Satisfaction with mental health services in older primary care patients. Am J Geriatr Psychiatry. 2006; 14:371-379.

40. Speer DC, Schneider MG. Mental health needs of older adults and primary care: opportunity for interdisciplinary geriatric team practice. Clin Psychol Sci Pract. 2003;21:85-101.

41. James LC. Integrating clinical psychology into primary care settings. J Clin Psychol. 2006;62:1207-1212.

42. Zivin K, Pfeiffer PN, Szymanski BR, et al. Initiation of primary care-mental health integration programs in the VA health system: associations with psychiatric diagnoses in primary care. Med Care. 2010;48:843-851.

43. Wang PS, Simon GE, Avorn J, et al. Telephone screening, outreach, and care management for depressed workers and impact on clinical and work productivity outcomes: a randomized controlled trial. JAMA. 2007;298:1401-1411.

44. Unutzer J, Katon W, Williams JW Jr, et al. Improving primary care for depression in late life: the design of a multicenter randomized trial. Med Care. 2001;39:785-799.

45. Kainz K. Barriers and enhancements to physician psychologist collaboration. Prof Res Pract. 2002;33:169-175.
46. Cummings NA, O’Donohue WT, Cummings JL. The financial dimension of integrated behavioral/primary care. J Clin Psychol Med Settings. 2009; 16:31-39.

47. Rosenthal MB, Dudley RA. Pay-for-performance: will the latest payment trend improve care? JAMA. 2007;297:740-744.

48. Mahar M. Money-driven medicine: the real reason health care costs so much. New York: Doubleday; 2006.

49. Greenberg JO, Dudley JC, Ferris TG. Engaging specialists in performance-incentive programs. N Engl J Med. 2010;362:1558-1560.

50. Stuart RB. Critical reappraisal of selected mental health programs. In: Hamerlynck LA, Acker LE, editors. Behavior modification and ideal mental health services. Calgary, AB: University of Calgary Press; 1969:5-100.

51. Marcus SC, Olfson M. National trends in the treatment for depression from 1998 to 2007. Arch Gen Psychiatry. 2010;67:1265-1273.

52. Paulose-Ram R, Safran MA, Jonas BS, Gu Q, Orwig D. Trends in psychotropic medication use among US adults. Pharmacoepidemiol Drug Saf. 2007;16:560-570.

53. Colenda CC, Mickus MA, Marcus SC, Tanielian TL, Pincus HA. Comparison of adult and geriatric psychiatric practice patterns: findings from the American Psychiatric Association's Practice Research Network. Am J Geriatr Psychiatry. 2002;10:609-617.

54. Thomas CP, Conrad P, Casler R, Goodman E. Trends in the use of psychotropic medications among adolescents, 1994 to 2001. Psychiatr Serv. 2006;57:63-69.

55. Wong IC, Murray ML, Camilleri-Novak D, Stephens P. Increased prescribing trends of paediatric psychotropic medications. Arch Dis Child. 2004;89:1131-1132.

56. Cummings NA, O'Donohue WT. Eleven blunders that cripple psychotherapy in America: a remedial unblundering. New York: Routledge; 2008.

57. Kravitz RL, Epstein RM, Feldman MD, et al. Influence of patients' requests for direct-to-consumer advertised antidepressants: a randomized controlled trial. JAMA. 2005;293:1995-2002.

58. Schiff GD, Galanter WL. Promoting more conservative prescribing. JAMA. 2009;301:865-867.

59. Van Schaik DJ, Klijn AF, van Hout HP, et al. Patients' preferences in the treatment of depressive disorder in primary care. Gen Hosp Psychiatry. 2004;26:184-189.

60. Dwight-Johnson M, Sherbourne CD, Liao D, Wells KB. Treatment preferences among depressed primary care patients. J Gen Intern Med. 2000;15:527-534.

61. Ebmeier KP, Donaghey C, Steele JD. Recent developments and current controversies in depression. Lancet. 2006;367:153-167.

62. Bray JH. Training primary care psychologists. J Clin Psychol Med Settings. 2004;11:101-107.

63. Thorpe KE, Zwarenstein M, Oxman AD, et al. A pragmatic-explanatory continuum indicator summary (PRECIS): a tool to help trial designers. CMAJ. 2009;180:E47-E57.
Psychology Research and Behavior Management

\section{Publish your work in this journal}

Psychology Research and Behavior Management is an international, peerreviewed, open access journal focusing on the science of psychology and its application in behavior management to develop improved outcomes in the clinical, educational, sports and business arenas. Specific topics covered include: Neuroscience, memory \& decision making; Behavior

\section{Dovepress}

modification \& management; Clinical applications; Business \& sports performance management; Social and developmental studies; Animal studies. The manuscript management system is completely online and includes a quick and fair peer-review system. Visit http://www.dovepress. com/testimonials.php to read real quotes from published authors. 